\title{
Nontraumatic Myelopathy in Malawi: A Prospective Study in an Area with High HIV Prevalence
}

\author{
Eduard E. Zijlstra, ${ }^{1 *}$ Jaap J. van Hellemond, ${ }^{2}$ Arthur D. Moes, ${ }^{3}$ Christa de Boer, ${ }^{4}$ Shelley A. Boeschoten, ${ }^{5}$ \\ Catharina E. M. van Blijswijk, ${ }^{6}$ Roos M. van der Vuurst de Vries, ${ }^{7}$ Peter A. B. Bailey, ${ }^{8}$ Sam Kampondeni, ${ }^{9}$ Lisette van Lieshout, ${ }^{10}$ \\ Saskia L. Smits, ${ }^{11}$ Juri Katchanov, ${ }^{1,12}$ Nyengo M. Mkandawire, ${ }^{13}$ and Camilla Rothe ${ }^{1,14}$ \\ ${ }^{1}$ Department of Medicine, College of Medicine, Blantyre, Malawi; ${ }^{2}$ Department of Medical Microbiology and Infectious Diseases, Erasmus \\ University Medical Center, Rotterdam, The Netherlands; ${ }^{3}$ Division of Nephrology, Department of Internal Medicine, Erasmus University Medical \\ Center, Rotterdam, The Netherlands; ${ }^{4}$ Infectious Disease Control, Municipal Health Service Zuid-Holland Zuid, Dordrecht, The Netherlands; \\ ${ }^{5}$ Erasmus University Medical Center - Sophia Children's Hospital, Rotterdam, The Netherlands; ${ }^{6}$ Vrije Universiteit Medisch Centrum (VUMC), \\ Amsterdam, The Netherlands; ${ }^{7}$ Department of Neurology, Erasmus University Medical Center, Rotterdam, The Netherlands; ${ }^{8}$ Gold Coast \\ University Hospital, Southport, Australia; ${ }^{9}$ Sam Kampondeni Clinic, Blantyre, Malawi; ${ }^{10}$ Department of Parasitology, Leiden University Medical \\ Centre, Leiden, The Netherlands; ${ }^{11}$ Viroclinics Biosciences BV, Rotterdam Science Tower, Rotterdam, The Netherlands; ${ }^{12}$ Department of \\ Hematology and Oncology, LMU University of Munich, Munich, Germany; ${ }^{13}$ Department of Surgery, College of Medicine, Blantyre, Malawi; \\ ${ }^{14}$ Division of Infectious Diseases and Tropical Medicine, University Hospital, LMU Munich, Munich, Germany
}

\begin{abstract}
Nontraumatic myelopathy causes severe morbidity and is not uncommon in Africa. Clinically, patients often present with paraplegia, and extrinsic cord compression and transverse myelitis are most common causes. Data on exact pathogenesis are scanty because of limitations in diagnostic methods. In Queen Elizabeth Central Hospital, Blantyre, Malawi, we recorded consecutive patients presenting with nontraumatic paraplegia for maximally 6 months between January and July 2010 and from March to December 2011. The diagnostic workup included imaging and examining blood, stool, urine, sputum, and cerebrospinal fluid (CSF) samples for infection. After discharge, additional diagnostic tests, including screening for virus infections, borreliosis, syphilis, and schistosomiasis, were carried out in the Netherlands. The clinical diagnosis was, thus, revised in retrospect with a more accurate final differential diagnosis. Of 58 patients included, the mean age was 41 years (range, 12-83 years) and the median time between onset and presentation was 18 days (range, $0-121$ days), and of 55 patients tested, 23 (42\%) were HIV positive. Spinal tuberculosis $(n=24,41 \%)$, tumors $(n=16,28 \%)$, and transverse myelitis $(n=6,10 \%)$ were most common; in six cases (10\%), no diagnosis could be made. The additional tests yielded evidence for CSF infection with Schistosoma, Treponema pallidum, Epstein-Barr virus (EBV), HHV-6, HIV, as well as a novel cyclovirus. The diagnosis of the cause of paraplegia is complex and requires access to an magnetic resonance imaging (MRI) scan and other diagnostic (molecular) tools to demonstrate infection. The major challenge is to confirm the role of detected pathogens in the pathophysiology and to design an effective and affordable diagnostic approach.
\end{abstract}

\section{INTRODUCTION}

Nontraumatic myelopathy is not uncommon in tropical medicine. Recent onset paraplegia is often the presenting clinical syndrome and may be caused by compressive disorders such as tumors or infections (abscesses and granulomatous disease) as well as non-compressive disorders such as transverse myelitis caused by infections that may include HIV, the human T-cell lymphotropic virus (HTLV-1), and syphilis. Schistosomiasis may cause myelopathy through both mechanisms.

In Africa, tuberculous spondylitis is common, particularly among HIV-infected individuals. The clinical manifestations are caused by destruction of vertebrae or by a paraspinal abscess. ${ }^{1-3}$ Schistosomiasis is endemic in many regions in Africa, and spinal cord schistosomiasis (SCS) has been well described in tourists and expatriates. ${ }^{4}$ It is usually caused by ectopic deposition of Schistosoma eggs in the spinal cord, and occasionally, adult worms have been found in the leptomeningeal veins. ${ }^{5,6}$ Compression of the spinal cord occurs because of granulomatous inflammation with prominent motor disturbance (most common presentation) or there may be necrosis of the myelum, resulting in a rapidly progressive transverse myelitis with a sharp sensory level (less common). ${ }^{7}$ Other causes include HTLV-1-associated myelopathy, malignancies (primary tumors of the spine or due to metastasis), degenerative disease, and (infective) myelitis. ${ }^{8,9}$

*Address correspondence to Eduard E. Zijlstra, Rotterdam Centre for Tropical Medicine, Bovenstraat 21, Rotterdam 3077 BB, The Netherlands. E-mail: e.e.zijlstra@roctm.com
Nontraumatic myelopathy is not uncommon among medical and surgical admissions at Queen Elizabeth Central Hospital (QECH), Blantyre, Malawi, which functions as a tertiary referral hospital in the southern region of Malawi. As diagnostic facilities are limited, the cause often remains unknown. In a study performed between 2000 and 2002 at $\mathrm{QECH}, 16$ of 33 patients with nontraumatic myelopathy were presumed to have SCS. ${ }^{10}$ In addition, HIV infection is common (24\% among urban adult population in Malawi, 70\% among medical admissions, and $20 \%$ among surgical admissions), and a sharp increase in tuberculosis has followed the introduction of HIV in the 1990s. ${ }^{11}$ The HIV infection has been implicated in various neurological syndromes as well, including myelopathy, but it is currently unknown to what extent HIV infection or associated viral infections may play a role. Similarly, there is no information on the contribution of benign or malignant tumors. According to the current practice at $\mathrm{QECH}$, all patients presenting with recent onset paraplegia are empirically treated for schistosomiasis with praziquantel.

We attempted to describe consecutive cases of recent onset nontraumatic paraplegia in the medical and surgical departments of QECH, to describe (groups of) diagnoses, and to provide recommendations for management.

\section{METHODS}

Between January and July 2010 and from March to December 2011, all consecutive patients in QECH, Blantyre, Malawi, with nontraumatic paraplegia of less than 6 months duration were studied. Clinical assessment was not different from routine practice; this included a detailed history and 
physical examination, in which the neurological findings were recorded using the standard neurological examination of spinal injury by the American Spinal Injury Association (ASIA) (http://asiaspinalinjury.org/wp-content/uploads/2016/02/International_Stds_ Diagram_Worksheet.pdf).

On discharge from the hospital, the neurological examination was repeated, and the ASIA score was recorded. Subsequently, the patients were referred to a rehabilitation clinic.

In addition, radiological imaging (chest X-ray, spinal X-ray, ultrasound, and MRI) were performed as appropriate.

Laboratory procedures at QECH. If indicated, an HIV test was obtained. Full blood and CD4 counts were carried out. Urine was tested for protein, glucose, and cells by dipstick rapid diagnostic tests.

Lumbar puncture was performed if clinically indicated, and routine analysis of cerebrospinal fluid (CSF) included protein and glucose levels; total leukocyte count and differentiation; and staining with Gram stain, Ziehl-Neelsen stain, and India ink.

Schistosomiasis was diagnosed by demonstration of Schistosoma eggs by microscopic examination of concentrated samples of urine (filtration) and stool (Kato-Katz method). Where possible, three consecutive stool samples and three consecutive urine samples were collected and examined per patient. Microscopy findings were scored as positive or negative; the number of eggs found was not recorded.

Although other helminthic infections were occasionally detected, these were not included in this report.

Radiological imaging. This included a chest or spinal X-ray and an ultrasound. The radiological examinations were not standardized but were at the discretion of the attending clinician who was guided by the clinical presentation, that is, the symptoms and signs at presentation. MRI scan services were restricted, and an MRI scan was only performed when clinically indicated. The MRI scans were reported by a radiologist (SK).

Surgical intervention. If indicated, the spinal lesion was surgically explored for drainage or removal of a mass. In some cases, specimens could be examined by a pathologist for histological diagnosis.

Additional laboratory procedures. Additional examinations were retrospectively performed in stored samples of serum, urine, stool, and CSF by the Erasmus University Medical Center, Department of Virology and Department of Medical Microbiology and Infectious Diseases, and the Leiden University Medical Center, Department of Parasitology, in the Netherlands.

The presence of DNA of Schistosoma species in frozenstored urine, stool, and CSF samples was performed by realtime polymerase chain reaction (PCR) as described for stool samples before. ${ }^{12}$ Only one stool and one urine sample per patient were analyzed by this PCR. Antibodies against Schistosoma species were determined as described before with minor modifications. ${ }^{13}$ The Schistosoma egg antigen ELISA test result was considered negative if $<100$ and equivocal if between 100 and 200 .

Intrathecal production of Schistosoma-specific antibodies was investigated as described before. ${ }^{14}$ The albumin index (albumin in CSF/albumin in serum) was used as a measure for integrity for the blood-brain barrier (BBB). If the albumin index was $<0.0090$, the BBB was assumed intact and intrathecal anti-Schistosoma IgG production could be examined by calculating the anti-egg versus anti-worm antibody index, which is defined as follows:

$$
\left(E_{c} / W_{c}\right) /\left(E_{s} / W_{s}\right)
$$

where $W_{c}=$ anti-worm $\operatorname{lgG}$ in CSF, Ws = anti-worm IgG in serum, Ec = anti-egg IgG in CSF, and Es = anti-egg IgG in serum.

If $>4$, the index indicates intrathecal schistosomiasis; if 2-4, the index is equivocal; and if $<2$, the index is negative. ${ }^{14}$

Borreliosis was diagnosed by quantitative determination of specific lgG in serum and CSF against recombinant antigens of Borrelia burgdorferi, using a commercially available chemiluminescence immunoassay (DiaSorin, Saluggia, Italy) that was performed according to the manufacturer's instructions.

Syphilis was diagnosed by a commercially available Treponema pallidum particle agglutination assay (TPPA) (Serodia, Fujirebio Inc., Tokyo, Japan). Serum and CSF were examined for the presence of specific antibodies against T. pallidum by serial dilution according to the manufacturers' instructions.

Tuberculosis was diagnosed according to the guidelines of the Malawi Tuberculosis Control Programme and included examination of sputum, lymph node aspirate, or pleural fluid specimens with Ziehl-Neelsen stain and radiological imaging. In addition, the presence of Mycobacterium tuberculosis in CSF was examined by a commercially available PCR assay according to the manufacturer's protocol (Hain Lifescience, Nehren, Germany).

Molecular virology screening. All samples were screened for the presence of viral pathogens by real-time (reversetranscriptase) PCRs with primers and probes used in a routine setting of Erasmus University Medical Center molecular viral diagnostics. ${ }^{15}$

For detection of HSV-1, HSV-2, CMV, EBV, VZV, HHV-6, $\mathrm{HHV}-8$, Enterovirus, Parechovirus, and JC virus in CSF samples, a semiautomatic workflow was including a Xiril-a (preextraction sample handling), MagNa Pure96 (nucleic acid extraction), Xiril-y (PCR-setup), LC480-II, and middleware software AuroraFlow (Roche Diagnostics, Almere, the Netherlands). Total nucleic acids were isolated using an input and output volume of $100 \mu \mathrm{L}$ and $300 \mu \mathrm{L}$, respectively. For the detection of chikungunya virus, HIV-1, HIV-2, hepatitis B virus (HBV), and HTLV-1 in serum, nucleic acids were extracted using MagNa PureLC (Roche Diagnostics, Almere, the Netherlands), with the High Pure 200 extraction protocol and the total nucleic acid isolation kit, with an input and output volume of $100 \mu \mathrm{L}$ and $200 \mu \mathrm{L}$, respectively. Because of the limited amount of sample volume, the samples had a final dilution of 6-60x compared with routinely analyzed samples in the diagnostic setting, depending on the available sample volume.

Input volumes for real time reverse transcription polymerase chain reaction (RT-PCR) assays for RNA viruses were $8 \mu \mathrm{L}$ for entero- and parechoviruses and $20 \mu \mathrm{L}$ for HIV-1, HIV-2, and chikungunya virus. Input volumes for real-time PCR assays for DNA viruses were $8 \mu \mathrm{L}$ (for EBV, CMV, VZV, HHV-6, HHV-8, and JC), $10 \mu \mathrm{L}$ (for HSV-1 and HSV-2), or $20 \mu \mathrm{L}$ (for HBV). The extracted RNA or DNA was amplified as described previously. ${ }^{15}$

For HTLV-1 infection, $5 \mu \mathrm{L}$ of RNA was used. The extracted RNA was converted to cDNA and amplified essentially as described, using primers P-tRNA (gamma $F$ ) (5'CADKTGGGGGCTCGTCCGGGAT-3) and POL-3 (delta R) (5'-GGCCTGGAGGCGYTCHRGTTTAAMGG-3'). ${ }^{16}$ The PCR 
TABLE 1

Baseline data of 58 patients enrolled with presumed nontraumatic paraplegia of less than 6 months duration according to HIV status

\begin{tabular}{|c|c|c|c|}
\hline & HIV positive $^{\star}$ & HIV negative $^{*}$ & HIV unknown \\
\hline Number & 23 & 32 & 3 \\
\hline Age mean, years (SD) & $38.3(10.7)$ & $41.3(20.5)$ & $58.3(20.7)$ \\
\hline Age range (years) & $16-57$ & $12-83$ & $36-77$ \\
\hline Male, number (\%) & $12(52)$ & $23(72)$ & $2(67)$ \\
\hline $\begin{array}{l}\text { Time between onset and presentation } \\
\text { (days), mean (SD) }\end{array}$ & $31(33)$ & $26(29)$ & $20(30)$ \\
\hline On antiretroviral therapy (ARV), number (\%) & $17(83)$ & NA & NA \\
\hline \multicolumn{2}{|l|}{$\begin{array}{l}\text { Time on ARVs (months), number of } \\
\text { patients (\%) }\end{array}$} & NA & NA \\
\hline $0-4$ & $2(12 \%)$ & & \\
\hline $5-8$ & $3(18 \%)$ & & \\
\hline $9-12$ & $1(6 \%)$ & & \\
\hline $13-24$ & $1(6 \%)$ & & \\
\hline$>24$ & $10(59 \%)$ & & \\
\hline \multicolumn{4}{|l|}{ Symptoms $(n=57)$} \\
\hline Fever & $11(19 \%)$ & $11(19 \%)$ & 0 \\
\hline Cough & $3(5 \%)$ & $3(5 \%)$ & 0 \\
\hline Abdominal pain & $6(11 \%)$ & $8(14 \%)$ & $2(4 \%)$ \\
\hline Weight loss & $9(16 \%)$ & $14(25 \%)$ & $1(2 \%)$ \\
\hline Back pain & $18(32 \%)$ & $24(42 \%)$ & $2(4 \%)$ \\
\hline Paresthesia & $23(40 \%)$ & $27(47 \%)$ & $3(5 \%)$ \\
\hline Weakness & $23(40 \%)$ & $29(51 \%)$ & $3(5 \%)$ \\
\hline Dysuria & $5(9 \%)$ & $4(7 \%)$ & $1(2 \%)$ \\
\hline Sphincter disturbance & $12(21 \%)$ & $16(28 \%)$ & $2(4 \%)$ \\
\hline \multicolumn{4}{|l|}{ Signs $(n=56)$} \\
\hline \multicolumn{4}{|l|}{$\begin{array}{l}\text { American Spinal Injury Association } \\
\text { score on admission, number (\% of } \\
\text { score A, B, C or D) }\end{array}$} \\
\hline $\begin{array}{l}\text { A (complete, no motor or sensory } \\
\text { function preserved in sacral } \\
\text { segments S4-S5) }\end{array}$ & $8(35 \%)$ & $13(41 \%)$ & $2(67 \%)$ \\
\hline $\begin{array}{l}\text { B (incomplete, sensory function } \\
\text { below the neurological level and } \\
\text { includes preserved in S4-S5) }\end{array}$ & $4(17 \%)$ & $4(13 \%)$ & $1(33 \%)$ \\
\hline $\begin{array}{l}\text { C (incomplete, motor function } \\
\text { preserved below the } \\
\text { neurological level, and } \\
\text { preserved below the level in } \\
\geq 50 \% \text { of muscles, with grade }<3 \text { ) }\end{array}$ & $6(26 \%)$ & $8(25 \%)$ & 0 \\
\hline $\begin{array}{l}\mathrm{D} \text { (incomplete, motor function } \\
\text { power preserved below the } \\
\text { neurological level, and } \\
\text { preserved below the level in } \\
\geq 50 \% \text { of muscles with } \geq \text { grade } 3 \text { ) }\end{array}$ & $5(21 \%)$ & $7(22 \%)$ & 0 \\
\hline \multicolumn{4}{|l|}{ Laboratory tests $(n=56)$} \\
\hline Hemoglobin, mg/dL (SD) & $12.2(3.2)$ & $11.7(3.0)$ & $13.2(1.1)$ \\
\hline $\begin{array}{l}\text { Total leukocyte count in cells } \times 10^{3} \text { / } \\
\mathrm{mm}^{3}(\mathrm{SD})\end{array}$ & $7.7(7.2)$ & $6.8(3.56)$ & $5.2(1.2)$ \\
\hline Platelets in cells $/ \mathrm{mm}^{3}$ (SD) & $314(119)$ & $277(155)$ & $289(120)$ \\
\hline CD4 count (range) in cells $/ \mathrm{mm}^{3}$ & 222 (157) 39-535 & NA & NA \\
\hline
\end{tabular}

* No statistically significant differences between HIV positive and HIV negative were observed.

temperature profile was 7 minutes at $95^{\circ} \mathrm{C}, 40$ cycles of 40 seconds at $95^{\circ} \mathrm{C}, 1$ minutes at $65^{\circ} \mathrm{C}$ and 2 minutes at $72^{\circ} \mathrm{C}$, and final extension 10 minutes at $72^{\circ} \mathrm{C}$.

A subset of samples was analyzed previously using a metagenomics approach in a virus discovery study. ${ }^{17}$

Clinical and final diagnosis. Based on the clinical assessment during admission, a most likely clinical diagnosis was made, and medical or surgical treatment was commenced as appropriate. After reviewing of the additional laboratory results, a most likely final (differential) diagnosis was made in retrospect.

Neuroschistosomiasis was diagnosed using a positive PCR test result in the CSF or on evidence of intrathecal anti- schistosomal antibody production. Neurosyphilis was diagnosed by using TPPA in serum and in CSF, with an intact BBB. Neuroborreliosis was diagnosed if evidence was found for intrathecal production of anti-Borrelia lgG with an intact BBB. Myelopathy associated with HSV-1, HSV-2, CMV, EBV, VZV, HHV-6, HHV-8, Enterovirus, Parechovirus, JC virus, and HTLV-1 was diagnosed on the presence of a positive PCR result in the CSF. M. tuberculosis infection of the central nervous system was confirmed by CSF analysis including Ziehl-Neelsen staining and PCR. Spinal tuberculosis was considered in case of a positive Ziehl-Neelsen stain of the sputum, and/or X-rays of the chest and spine, in combination with an MRI suggestive of tuberculosis, or a total leukocyte 
count and differential cell count, and protein and glucose levels in the CSF suggestive of tuberculosis. Tumors or metastases were either diagnosed by histological examination of a biopsy or material obtained during surgery.

Data collection and analysis. The data were entered on a patient record form and subsequently analyzed using SPSS 24. Standard tabulation and descriptive methods, such as means, were used for descriptive purposes. Means were compared using independent sample $t$-test and proportions by using chi-squared test. A significance level of $P<0.05$ was used. Logistic regression was used to explore whether combinations of variables were associated with common final diagnoses.

Ethical approval and informed consent. The study received approval from the College of Medicine Research and Ethics Committee and College of Medicine, Blantyre, Malawi. Informed consent was obtained from all patients.

\section{RESULTS}

The total number of patients enrolled was 58, of which 37 (64\%) were male. The mean age was 41.0 years (SD, 17.5; range, 12-83 years). The median time between the onset of symptoms and presentation was 18 days (range, $0-121$ days). The presence of HIV antibodies was examined in 55 patients, of which $23(42 \%)$ tested positive. Baseline characteristics of the included patients are summarized in Table 1.

According to the ASIA scale at admission and at discharge, 12 patients improved, whereas three deteriorated and 32 had stable disease; for 11 patients, the ASIA score at discharge was not recorded. Eight patients died during hospitalization.

The mean hemoglobin was $12.0 \mathrm{mg} / \mathrm{dL}(\mathrm{SD}, 3.1$; range, 4.0-20.5), the total leukocyte count was $7.1 \times 10^{3}$ cells $/ \mathrm{mm}^{3}$ (SD, 5.3; range, 1.9-29.2), and the mean platelet count was $293 \times 10^{3}$ cells $/ \mathrm{mm}^{3}$ (SD, 139; range, 19-640).

In 39 patients, a lumbar puncture was performed, and in eight of these, an increased leukocyte count $\geq 5$ cells $/ \mathrm{mm}^{3}$ was found (range, 8-80). The CSF protein levels were raised in 28 patients, with mean 3.6 (SD 2.4) and in the range of $0.49-8.18 \mathrm{mg} / \mathrm{dL}$ (normal 0.15-0.40 mg/dL).

In Supplemental Table 1, clinical, laboratory, radiological, and pathological information of the 58 cases are shown with the clinical diagnosis made during admission and the most likely final (differential) diagnosis in retrospect, after analysis of additional laboratory procedures.

Detection of schistosomiasis. In 57 of the 58 included patients, an attempt to diagnose schistosomiasis could be made by either microscopy or PCR analysis of urine $(n=57,98 \%)$ or stool $(n=47,81 \%)$.

Three consecutive urine samples could be examined by microscopy in 46 patients (79\%). For stool, this was in 24 patients $(41 \%)$. Schistosoma eggs were seen in seven patients (12\%). In six patients, eggs were demonstrated in stool: in five cases, Schistosoma mansoni was found, and in one case, Schistosoma haematobium was found. In three patients, S. haematobium eggs were demonstrated in urine. In two patients, eggs were found both in stool and urine samples; one had double infection with S. mansoni and S. haematobium, and one had $S$. haematobium eggs in both sample types. The PCR analysis demonstrated Schistosoma spp. DNA in another seven patients ( $n=2$ in stool and $n=5$ in urine). The presence of Schistosoma eggs observed in three patients $(n=$ 2 in stool and $n=1$ in urine) could not be confirmed by PCR. In total, 14 of the 57 examined patients (25\%) were microscopy and/or PCR positive, so these patients were considered to have active schistosomiasis.

No DNA of Schistosoma spp. could be detected in any of 39 (67\%) tested CSF samples.

Of 52 serum samples examined, 13 patients (25\%) were found positive for antibodies against Schistosoma worm or egg antigens; 34 patients (65\%) were negative, and in five patients (10\%), the result was equivocal. In the CSF of 17 patients examined, using the egg-worm index, two patients had a score of $>4$, whereas in another two patients, the result was equivocal (score 2-4). Hence, neuroschistosomiasis was included in the final differential diagnosis in these four cases (Supplemental Table 1).

Detection of virus infections. Of 55 serum samples tested for HIV at presentation in QECH, using routine screening with two rapid antibody tests, $23(40 \%)$ were positive, with a mean CD4 count of 221 cells $/ \mathrm{mm}^{3}$ (range, 39-535); in 67\%, the CD4 count was $<200$ cells $/ \mathrm{mm}^{3}$

The additional laboratory investigations retrospectively performed in the Netherlands were carried out on 50 serum samples and $35 \mathrm{CSF}$ samples. Using routine real-time RTPCR assays, HIV-1 was detected in 11 of 50 (22\%) of serum samples and in nine of 35 CSF samples (26\%); all but one had a CD4 count $<200 \mathrm{~mm}^{3}$. In eight patients, these findings correlated in serum and CSF; in one patient, there was lack of serum. In seven of nine who were positive for HIV in CSF, HIV-1 was the only virus detected; in one HIV-1-positive patient, EBV also was demonstrated in CSF as well as HBV in serum. Another HIV-1-positive patient was also positive for human cyclovirus.

Cyclovirus was detected using a metagenomics approach in $15 \%$ and $10 \%$ of serum samples, and CSF samples, respectively. ${ }^{17}$

Hepatitis B virus was detected in the serum of six patients, two of whom had hepatocellular carcinoma. In one HIV-1negative patient, HHV-6 was detected in CSF.

No evidence was found for VZV, CMV, HSV-1, HSV-2, enterovirus (including polio), parechovirus, JCV, or HTLV-1 infections (only CSF samples examined) or HIV-2 and chikungunya virus (both CSF and serum samples examined) (Supplemental Table 1).

Detection of bacterial infections. No positive ZiehlNeelsen stain was found in the CSF samples examined.

All 41 examined CSF samples tested for mycobacterial infection by PCR were negative.

Antibodies against $T$. pallidum were detected by using TPPA in eight of 53 (15\%) examined serum samples. In four of those patients, the CSF was enriched for T. pallidum-specific IgG, suggesting neurosyphilis that was, therefore, considered to be in the differential diagnosis in these four cases (Supplemental Table 1).

None of the 53 examined CSF samples were enriched in Borrelia-specific lgG.

Table 2 shows the distribution of symptoms, ASIA score, and main laboratory findings at presentation according to the most likely final differential diagnosis.

Using multivariate logistic regression, HIV status was found predictive of the diagnosis of spinal tuberculosis $(P=0.018)$. The total white cell count (TWC) and ASIA score were not predictive of this diagnosis. 


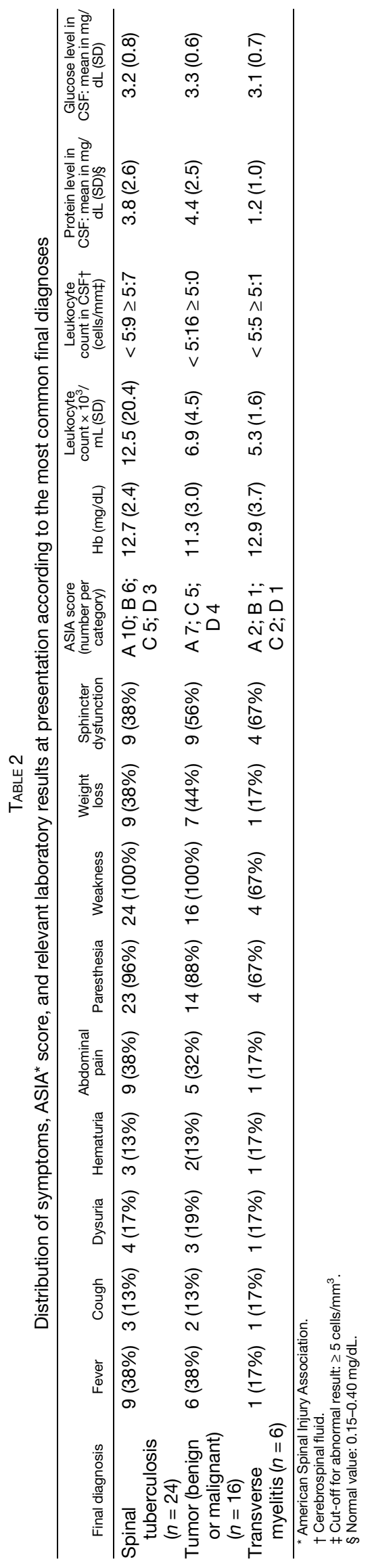

In Table 3, the most likely final diagnoses are summarized in categories according to HIV status. Of 58 patients studied, myelopathy was diagnosed in 46 (79\%), whereas in others, radiculopathy by disk herniation (two patients, 3\%) or polyneuropathy (4 patients, $7 \%$ ) was considered likely. In four patients (12\%), the diagnosis remained entirely unknown, whereas in two patients, the most likely primary differential diagnosis was not established.

\section{DISCUSSION}

This study shows the diversity of differential diagnoses and the challenges in making a firm final diagnosis of the cause of paraplegia in a resource-limited setting. In 10 patients (17\%), the clinical diagnosis could be confirmed; in eight cases, confirmation was possible as surgery was indicated (laminectomy, drainage, or tumor excision with subsequent histological examination of the surgical specimen), and in two other cases with transverse myelitis, EBV and HSV-2 virus were found respectively, in the CSF by PCR, in the absence of another diagnosis.

In 23 patients (41\%), spinal tuberculosis was considered the most likely final diagnosis. A positive HIV test appeared to be a predictor for spinal tuberculosis. However, the CSF of all patients tested for mycobacteria by PCR was negative. This may be caused by the limited sensitivity of this test of $<40 \%$ that may be negatively influenced by the small volume CSF used and the freezing of the sample before analysis in this study. ${ }^{18}$ Of the 23 patients with presumed spinal tuberculosis, tuberculosis (TB) was considered likely in 16 patients because of clinical features (draining abscess, gibbus, abnormal chest X-ray showing pleural effusion, or miliary TB) or spinal X-ray (destruction of vertebrae and paraspinal mass). In the other seven patients, TB was the most likely diagnosis based on only a suggestive MRI result. The value of the MRI has been emphasized and may distinguish between spondylitis and non-spondylitis tuberculosis. ${ }^{2}$

Spinal tuberculosis was more common in HIV-positive patients (14 [61\%] compared with HIV-negative patients (9 [28\%]), and HIV infection was the only predictor found. This is in agreement with a study from South Africa where spinal tuberculosis was diagnosed in $50 \%$ and $11 \%$ of patients who were HIV positive and negative, respectively. ${ }^{3}$

The prevalence of schistosomiasis in the patients studied was high (25\%), and in half of these patients, an active infection could only be demonstrated by a positive PCR result for stool or urine specimens. Sampling of urine and stool proved difficult in this setting, and the contribution of microscopically diagnosed cases may, therefore, have been underestimated. No conclusive evidence for neuroschistosomiasis could be found, as all CSF samples tested were negative in the PCR. Detection of Schistosoma DNA in CSF is a sensitive method for detection of neuroschistosomiasis if DNA can be extracted from a large volume of CSF. ${ }^{19}$ Because of the limited amount of available material, DNA could only be isolated from $200 \mu \mathrm{L}$ of CSF. On the other hand, in $25 \%$ of the patients, antibodies against Schistosoma spp. were found, of which two patients had evidence for intrathecal production of schistosomespecific antibodies (and two with equivocal results). In these cases, neuroschistosomiasis infection was thought to be in the differential diagnosis of nontraumatic myelopathy. This low number of possible neuroschistosomiasis cases was 
TABLE 3

Final diagnosis categories

\begin{tabular}{|c|c|c|c|c|c|}
\hline Diagnosis & Number (\% of 58 patients examined) & Mean age, years (range) & HIV positive $(n=23)$ & HIV negative $(n=32)$ & HIV unknown $(n=3)$ \\
\hline Spinal tuberculosis & $24(41 \%)$ & $38(12-65)$ & $14(61 \%)$ & $9(28 \%)$ & $1(33 \%)$ \\
\hline Malignancy & $14(24 \%)$ & $51(25-83)$ & 0 & $12(38 \%)$ & $2(67 \%)$ \\
\hline Prostate & 2 & - & - & - & 1 \\
\hline Liver & 2 & - & - & - & - \\
\hline Bladder & 1 & - & - & - & - \\
\hline Burkitt's lymphoma & 1 & - & - & - & - \\
\hline Plasmocytoma & 1 & - & - & - & 1 \\
\hline Other/not known & 7 & - & - & - & - \\
\hline Transverse myelitis & $6(10 \%)$ & $41(23-61)$ & $2(9 \%)$ & $4(13 \%)$ & 0 \\
\hline Schistosomiasis & 2 & - & - & 2 & - \\
\hline Schistosomiasis or syphilis & 1 & - & - & 1 & - \\
\hline EBV & 1 & - & 1 & 0 & - \\
\hline HSV -2 & 1 & - & 0 & 1 & - \\
\hline HIV & 1 & - & 1 & 0 & - \\
\hline Benign tumors & $2(3 \%)$ & $25(23-28)$ & 0 & $2(6 \%)$ & 0 \\
\hline Schwannoma & 1 & - & - & 1 & - \\
\hline Hemangioma & 1 & - & - & 1 & - \\
\hline Disk prolapse & $2(3 \%)$ & - & $1(4 \%)$ & $1(3 \%)$ & 0 \\
\hline Polyneuropathy & $4(7 \%)$ & - & $3(13 \%)$ & $1(3 \%)$ & 0 \\
\hline Unknown & $6(10 \%)$ & - & $3(13 \%)$ & $3(9 \%)$ & 0 \\
\hline
\end{tabular}

First listed diagnosis in differential diagnosis, according to HIV status.

unexpected, given earlier reports in which schistosomiasis was estimated to cause half the cases of nontraumatic myelopathy cases in Malawi. ${ }^{10}$ The reason for this difference is unclear. Recent reports suggest that the incidence of schistosomiasis in Malawi has not declined, in view of poor control of schistosomiasis and the reported high morbidity due to schistosomiasis among the local population and continued risk of infection in travelers returning from Malawi. ${ }^{20-22}$ The increased awareness of Schistosoma infection as a cause of paraplegia and empirical treatment with praziquantel in local health facilities may have caused improvement or cure rates, possibly resulting in early treatment and/or a referral bias. A highly sensitive and easy to use point-of-care test to demonstrate an active Schistosoma infection would facilitate any presumptive diagnosis of neuroschistosomiasis. ${ }^{16}$ The performance of such a test and its contribution to the differential diagnosis of nontraumatic myelopathy should be evaluated in a prospective study.

Among viral infections, HIV infection was most common with a high prevalence in CSF samples examined $(n=9 ; 26 \%)$. Most of these patients were profoundly immunosuppressed. Viral infection with EBV or HIV was considered the cause of transverse myelitis in two patients (based on virus detection by PCR). In another patient, VZV infection was considered based on the recent clinical presentation with herpes zoster. In another study in Malawi in which 14 patients with viral meningitis were described, EBV has been found in $50 \%$ of cases. In that study, CMV and HSV-1 were also found and all patients were HIV positive. ${ }^{23}$ The high prevalence of cyclovirus in CSF of patients included in this study was surprising. The clinical relevance is unclear, but this virus has been implicated in acute nervous system infections in Vietnam. ${ }^{17,24}$ Further study is needed in other patient groups including healthy controls before conclusions can be drawn concerning the pathogenicity of this virus. No cases of HTLV-1-associated myelopathy could be demonstrated, although both HTLV-1 and HTLV-2 circulate in Malawi and have been detected among mothers and children. ${ }^{25}$
Evidence for neurosyphilis was found in four patients and, thus, contributed to the differential diagnosis.

Limitations of the study. This study followed the current clinical practice regarding diagnosis and management of patients presenting with non-traumatic myelopathy. Given the limited resources a practical approach was often followed, and a confirmed histological diagnosis was only made in a minority of patients, restricted to those in whom surgery was indicated. MRI imaging is not unrestricted in our setting but contributed considerably in those in whom the diagnosis was largely unclear. Invasive diagnostic procedures aimed at spinal lesions carry risk and this should be balanced against a thorough clinical assessment including imaging and subsequent therapeutic approach.

This study shows the predominance of tuberculosis as the cause of nontraumatic myelopathy, largely driven by HIV infection. In most cases, spinal tuberculosis can already be expected on evidence of tuberculosis found elsewhere (sputum examination, chest $\mathrm{X}$-ray, and lymph node aspiration). The MRI imaging may provide strong support for tuberculosis. Recently, the use of the Xpert MTB/RIF assay in urine was reported as an aid to the diagnosis of tuberculous spondylitis. $^{26}$ Starting anti-tuberculous treatment based on these findings seems justified in the absence of other diagnostic possibilities. In patients with a mass that compresses the cord, surgical intervention is necessary. This shows that paraplegia patients should be assessed in a multidisciplinary team by internists or pediatricians, respectively, and orthopedic surgeons to determine the best approach to diagnosis and management.

Although only a few potential cases of SCS were identified, empirical treatment with praziquantel seems still justified in high prevalence areas, as treatment is safe and cheap.

Paraplegia is a devastating neglected (tropical) syndrome for which a minimum of diagnostic tools including MRI scanning should be available to limit prolonged and irreversible morbidity as the result of misdiagnosis, exposure to inappropriate drugs, and invasive procedures. 
Received March 15, 2019. Accepted for publication November 5, 2019.

Published online December 12, 2019.

Note: Supplemental table appears at www.ajtmh.org.

Acknowledgment: We are grateful to Nico Nagelkerke for statistical advice.

Authors' addresses: Eduard E Zijlstra, Department of Medicine, College of Medicine, Blantyre, Malawi, and Rotterdam Centre for Tropical Medicine, The Netherlands, E-mails: e.e.zijlstra@roctm.com. Jaap J. van Hellemond, Department of Medical Microbiology and Infectious Diseases, Erasmus University Medical Center, Rotterdam, The Netherlands, E-mail: j.vanhellemond@erasmusmc.nl. Arthur D. Moes, Division of Nephrology, Department of Internal Medicine, Erasmus University Medical Center, Rotterdam, The Netherlands, E-mail: arthurmoes@hotmail.com. Christa de Boer, Infectious Disease Control, Municipal Health Service Zuid-Holland Zuid, Dordrecht, The Netherlands, E-mail: c.de.boer@dienstgezondheidjeugd.nl. Shelley A. Boeschoten, Erasmus University Medical Center - Sophia Children's Hospital, Rotterdam, The Netherlands, E-mail: s.boeschoten@ erasmusmc.nl. Catharina E. M. van Blijswijk, VUMC, Amsterdam, The Netherlands, E-mail: c.vanblijswijk@vumc.nl. Roos M. van der Vuurst de Vries, Department of Neurology, Erasmus University Medical Center, Rotterdam, The Netherlands, E-mail: r.vandervuurstdevries@erasmusmc.nl. Peter AB Bailey, Gold Coast University Hospital, Southport, Australia, E-mail: pabbailey@ gmail.com. Sam Kampondeni, Sam Kampondeni Clinic, Blantyre, Malawi, E-mail: skampo@gmail.com. Lisette van Lieshout, Leiden University Medical Center, Leiden, The Netherlands, E-mail: Ivanlieshout@lumc.nl. Saskia L. Smits, Viroclinics Biosciences BV, Rotterdam, The Netherlands, E-mail: smits@viroclinics.com. Juri Katchanov, Department of Medicine, College of Medicine, Blantyre, Malawi, and Department of Hematology and Oncology, LMU University of Munich, Munich, Germany, E-mail: juri.katchanov@charite.de. Nyengo M. Mkandawire, Department of Surgery, College of Medicine, Blantyre, Malawi, E-mail: nmkandawire@medcol.mw. Camilla Rothe, Department of Medicine, College of Medicine, Chichiri, Blantyre, Malawi, and Division of Infectious Diseases and Tropical Medicine, University Hospital, LMU Munich, Munich, Germany, E-mail: camilla.rothe@web.de.

\section{REFERENCES}

1. Draulans N, Kiekens C, Roels E, Peers K, 2011. Etiology of spinal cord injuries in sub-Saharan Africa. Spinal Cord 49: 1148-1154.

2. Candy S, Chang G, Andronikou S, 2014. Acute myelopathy or cauda equina syndrome in HIV-positive adults in a tuberculosis endemic setting: MRI, clinical, and pathologic findings. AJNR Am J Neuroradio/ 35: 1634-1641.

3. Modi G, Ranchhod J, Hari K, Mochan A, Modi M, 2011. Nontraumatic myelopathy at the Chris Hani Baragwanath Hospital, South Africa-the influence of HIV. QJM 104: 697-703.

4. CDC, 1993. Schistosomiasis in U.S. Peace Corps VolunteersMalawi, 1992. MMWR Morb Mortal Wkly Rep 42: 565-570.

5. Pittella JE, 1997. Neuroschistosomiasis. Brain Pathol 7: 649-662.

6. Ferrari TC, Moreira PR, 2011. Neuroschistosomiasis: clinical symptoms and pathogenesis. Lancet Neurol 10: 853-864.

7. Haribhai HC, Bhigjee Al, Bill PL, Pammenter MD, Modi G, Hoffmann M, Kelbe C, Becker P, 1991. Spinal cord schistosomiasis. A clinical, laboratory and radiological study, with a note on therapeutic aspects. Brain 114: 709-726.

8. Bhigjee AI, Wiley CA, Wachsman W, Amenomori T, Pirie D, Bill PL, Windsor I, 1991. HTLV-I-associated myelopathy: clinicopathologic correlation with localization of provirus to spinal cord. Neurology 41: 1990-1992.

9. Touze E, Gessain A, Lyon-Caen O, Gout O, 1996. Tropical spastic paraparesis/HTLV-I-associated myelopathy in Europe and in Africa: clinical and epidemiologic aspects. J Acquir Immune Defic Syndr Hum Retrovirol 13 (Suppl 1): S38-S45.

10. Naus CW, Chipwete J, Visser LG, Zijlstra EE, van Lieshout L, 2003. The contribution made by Schistosoma infection to non- traumatic disorders of the spinal cord in Malawi. Ann Trop Med Parasitol 97: 711-721.

11. Lewis DK, Callaghan M, Phiri K, Chipwete J, Kublin JG, Borgstein E, Zijlstra EE, 2003. Prevalence and indicators of HIV and AIDS among adults admitted to medical and surgical wards in Blantyre, Malawi. Trans R Soc Trop Med Hyg 97: 91-96.

12. Meurs L, Brienen E, Mbow M, Ochola EA, Mboup S, Karanja DM, Secor WE, Polman K, van Lieshout L, 2015. Is PCR the next reference standard for the diagnosis of schistosoma in stool? A comparison with microscopy in Senegal and Kenya. PLoS Negl Trop Dis 9: e0003959.

13. Van Gool T, Vetter H, Vervoort T, Doenhoff MJ, Wetsteyn J, Overbosch D, 2002. Serodiagnosis of imported schistosomiasis by a combination of a commercial indirect hemagglutination test with Schistosoma mansoni adult worm antigens and an enzyme-linked immunosorbent assay with $S$. mansoni egg antigens. J Clin Microbiol 40: 3432-3437.

14. de Jongste $\mathrm{AH}$, Tilanus $\mathrm{AM}$, Bax $\mathrm{H}$, Willems $\mathrm{MH}$, van der Feltz $\mathrm{M}$, van Hellemond JJ, 2010. New insights in diagnosing Schistosoma myelopathy. $J$ Infect 60: 244-247.

15. Wilson S, Jones FM, van Dam GJ, Corstjens PL, Riveau G, Fitzsimmons CM, Sacko M, Vennervald BJ, Dunne DW, 2014. Human Schistosoma haematobium antifecundity immunity is dependent on transmission intensity and associated with immunoglobulin G1 to worm-derived antigens. J Infect Dis 210: 2009-2016.

16. Corstjens PL et al., 2014. Tools for diagnosis, monitoring and screening of Schistosoma infections utilizing lateral-flow based assays and upconverting phosphor labels. Parasitology 141: 1841-1855.

17. Smits SL, Zijlstra EE, van Hellemond JJ, Schapendonk CM, Bodewes R, Schurch AC, Haagmans BL, Osterhaus AD, 2013. Novel cyclovirus in human cerebrospinal fluid, Malawi, 2010-2011. Emerg Infect Dis 19. Available at: https://doi.org/ 10.3201/eid1909.130404.

18. Thwaites GE, Caws M, Chau TT, Dung NT, Campbell JI, Phu NH, Hien TT, White NJ, Farrar JJ, 2004. Comparison of conventional bacteriology with nucleic acid amplification (amplified Mycobacterium direct test) for diagnosis of tuberculous meningitis before and after inception of antituberculosis chemotherapy. J Clin Microbiol 42: 996-1002.

19. Harter G, Frickmann H, Zenk S, Wichmann D, Ammann B, Kern P, Fleischer B, Tannich E, Poppert S, 2014. Diagnosis of neuroschistosomiasis by antibody specificity index and semiquantitative real-time PCR from cerebrospinal fluid and serum. J Med Microbiol 63: 309-312.

20. Makaula P, Sadalaki JR, Muula AS, Kayuni S, Jemu S, Bloch P, 2014. Schistosomiasis in Malawi: a systematic review. Parasit Vectors 7: 570.

21. Mtethiwa AH, Nkwengulila G, Bakuza J, Sikawa D, Kazembe A, 2015. Extent of morbidity associated with schistosomiasis infection in Malawi: a review paper. Infect Dis Poverty 4: 25.

22. Moore E, Doherty JF, 2005. Schistosomiasis among travellers returning from Malawi: a common occurrence. QJM 98: 69-70.

23. Benjamin LA et al., 2013. Detection of herpes viruses in the cerebrospinal fluid of adults with suspected viral meningitis in Malawi. Infection 41: 27-31.

24. Tan le $V$ et al., 2013. Identification of a new cyclovirus in cerebrospinal fluid of patients with acute central nervous system infections. MBio 4: e00231-13.

25. Fox JM, Mutalima N, Molyneux E, Carpenter LM, Taylor GP, Bland M, Newton R, Martin F, 2016. Seroprevalence of HTLV-1 and HTLV-2 amongst mothers and children in Malawi within the context of a systematic review and meta-analysis of HTLV seroprevalence in Africa. Trop Med Int Health 21: 312-324.

26. Sikalengo G, Ramirez A, Faini D, Mwamelo K, Battegay M, Jugheli L, Hatz C, Reither K, Letang E, 2016. Tuberculous spondylitis diagnosed through Xpert MTB/RIF assay in urine: a case report. BMC Infect Dis 16: 514. 